\title{
Precision measurement of arrival times in an EAS by GRAPES-3 experiment
}

\author{
V.B. Jhansi ${ }^{* a b}$, S. Ahmad ${ }^{a c}$, K.P. Arunbabu ${ }^{a b}$, A. Chandra ${ }^{a b}$, S.R. Dugad ${ }^{a b}$, S.K. \\ Gupta $^{a b}$, B. Hariharan ${ }^{a b}$, Y. Hayashi ${ }^{a d}$, P. Jagadeesan ${ }^{a b}$, A. Jain ${ }^{a b}$, S. Kawakami ${ }^{a d}$, H. \\ Kojima $^{a e}$, P.K. Mohanty ${ }^{a b}$, S.D. Morris ${ }^{a b}$, P.K.Nayak ${ }^{a b}$, A. Oshima ${ }^{a f}$, B.S. Rao ${ }^{a b}$, L.V. \\ Reddy $^{a b}$, S. Shibata ${ }^{a f}$, M.Zuberi $^{a b}$ \\ ${ }^{a}$ The GRAPES-3 Experiment, Cosmic Ray Laboratory, Raj Bhavan, Ooty 643001, India \\ ${ }^{b}$ Tata Institute of Fundamental Research, Mumbai 400005, India \\ ${ }^{c}$ Aligarh Muslim Univeristy, Aligarh 202002, India \\ ${ }^{d}$ Graduate School of Science, Osaka City University, Osaka 558-8585, Japan \\ ${ }^{e}$ Faculty of Engineering, Aichi Insitute of Technology, Toyota City, Aichi 470-0392, Japan \\ ${ }^{f}$ College of Engineering, Chubu University, Kasugai, Aichi 487-8501, Japan
}

E-mail: jhansi1990@gmail.com

\begin{abstract}
In the GRAPES-3 experiment, consisting of an array of $\sim 400$ scintillator detectors, the arrival direction of the shower is determined from the relative arrival times of particles at different detectors. The fixed arrival time of the signal from the detector to the measuring device, referred as time offset, is crucial for an accurate measurement of shower direction. In the older method the time offset of various detectors was measured with respect to a common detector. But this method proved to be ineffective since it took a long time ( $\sim 40$ days) to complete one round of measurements. However, the time offsets vary with temperature due to change in the propagation delay in signal cables. Hence, a technique was devised to determine the time offsets on an hourly basis by using the shower data. In this method, the time offset between two neighbouring detectors was determined from the distribution of their relative arrival times. A random walk method was used to effectively determine the time offset with respect to a common detector. The accuracy of the new method was validated by using both the simulations, and EAS data which will be presented at the conference.
\end{abstract}

35th International Cosmic Ray Conference - ICRC2017

10-20 July, 2017

Bexco, Busan, Korea

*Speaker. 


\section{Introduction}

The GRAPES-3 shower array consists of about 400 plastic scintillators, each of area $1 \mathrm{~m}^{2}$, arranged in the form of hexagonal rings with $8 \mathrm{~m}$ seperation between adjacent detectors. Details of the detector, trigger and electronics are written elsewhere [1] [2]. Each detector is designed to measure the number of extensive air shower(EAS) particles passing through it, and their arrival times. For these timing measurements, the signal obtained from the photomultiplier tube(PMT) of each detector is transferred to the control room by a $230 \mathrm{~m}$ long co-axial cable, which is then amplified, discriminated and finally digitized by the TDCs(time-to-digital convertors) .

When a shower falls on the array, the TDC registers the time of arrival of shower particles with respect to time of arrival of trigger signal. However, the time measured in each detector is the sum of arrival times of particles and the time taken for the signal to be transferred from the detector to the TDC. The quantity mentioned later, referred to as time offset depends on several factors such as timing response of detector, propagation delay in signal cable and electronics such as amplifier discriminator etc. It varies significantly from detector to detector and it also varies with ambient conditions such as temperature. Determination of time offset is very crucial as the arrival direction of the shower is obtained by fitting a plane to the relative arrival times of shower front at different detectors.

The conventional method of time offset measurement at GRAPES-3 is made using a muon telescope which has to be moved manually from one detector to the other. Using this muon telescope, the data is taken for about 45 minutes for each detector, and hence only 8-10 detectors are calibrated per day. The next calibration of same detector occurs after about 40 days. Therefore short time variation due to temperature or any other reason cannot be taken care using this method. The new method developed using high statistics shower data enables the hourly determination of time offsets.

\section{Method}

Firstly, the difference between measured arrival times $\Delta \mathrm{t}$ between two neighbouring detectors is calculated for each shower. Here $\Delta \mathrm{t}$ can be factorised as follows.

$$
\begin{aligned}
& \Delta t=t_{\text {measured }}^{i}-t_{\text {measured }}^{j} \\
& =\left(t_{\text {sh }}^{i}+t_{\text {offset }}^{i}\right)-\left(t_{\text {sh }}^{j}+t_{\text {offset }}^{j}\right) \\
& =\left(t_{\text {sh }}^{i}-t_{\text {sh }}^{j}\right)+\left(t_{\text {offset }}^{i}-t_{\text {offset }}^{j}\right)
\end{aligned}
$$

Here $t_{s h}^{i}$ and $t_{s h}^{j}$ are the arrival times of shower front on detectors $\mathrm{i}$ and $\mathrm{j}$ whereas $t_{\text {offset }}^{i}$ and $t_{\text {offset }}^{j}$ corresponding time offsets.

Now the centroid of the $\Delta \mathrm{t}$ distribution(say $(\Delta t)_{p}$ ) gives the TDC offset between the detector $\mathrm{i}$ and $\mathrm{j}$ and the term $t_{s h}^{i}-t_{s h}^{j}$ is responsible for width of distribution

$$
t_{s h}^{i}-t_{s h}^{j}=\frac{d_{i j} \sin (\theta)}{c}
$$

Here $d_{i j}$ is the distance between two detectors and $\theta$ is the zenith angle of the shower. From the above formula, it is clear that the width of the distribution increases with distance between 
the detectors. In addition, when the distance between two neighbouring detectors increase, the probability that the shower hits both the detectors reduces. For these two reasons, we decided to calculate the relative time offsets of every neighbouring pair in the array. It has to be noted that the GRAPES-3 array is not flat and hence effective $\Delta t$ (in ns) is calculated as $\Delta t+\Delta z \times 3.33$ where $\mathrm{z}$ corresponds to the $\mathrm{z}$ co-ordintes of the detectors(in $\mathrm{m}$ ).(This formula is accurate only for vertical showers. But the zenith angle of the shower is an unknown parameter before reconscruction. Hence we use the above mentioned formula as an approximation.)

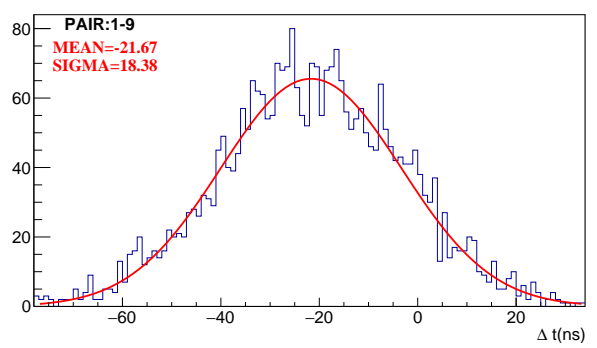

Figure 1: $\Delta t$ distribution of neighbouring detectors

However, it is essential to calculate the TDC offset of every detector with respect to a common detector. For this purpose, we used the random walk method. The method can be descibed as follows. Every detector in the array has sixteen neighbours. Starting with the reference detector, its time offset with respect to any of its sixteen neighbours has to be calculated for which an integer random number in the range of 1-16 is generated. Depending on the random number generated, any one of its neighbours is selected, and their relative time offset is measured. Now random numbers are produced again in order to select the neighbours of the previously selected detector. This procedure is continued till the target detector is reached, or else it will be discontinued if the boundary of the array is reached or if the destination detector is not reached in some specific number of steps. For each step, keep on adding the $(\Delta t)_{p}$ values measured directly from shower data to finally obtain the effective $(\Delta t)_{p}$ between reference and destination detector. Now if the process is repeated, the effective $(\Delta t)_{p}$ is calculated again but by following a different path(because of the usage of random number). Thus, by repeating the process, effective $(\Delta t)_{p}$ is calculated for multiple number of paths (In order to optimize compuation time, we have fixed the total number of paths to be 1000) and their mean value gives the precise value of time offset between the two detectors. Similarly the TDC offset of every detector is calculated with respect to the same reference detector.

The above technique can be illustrated with an example. Let D17 be the reference detector. We seek to determine the time offsets of all the detectors with respect to this common detector. Now we have to determine the time offset of D32 with respect to D17.D32 can be reached from D17 in a path passing through the nearest neighbours in certain number of steps, wherein each neighbour is selected using random numbers. This can be written mathematically as

$$
(\Delta t)_{p}^{17-32}=(\Delta t)_{p}^{17-56}+(\Delta t)_{p}^{56-35}+(\Delta t)_{p}^{35-88}+(\Delta t)_{p}^{88-57}+\ldots \ldots \ldots \ldots+(\Delta t)_{p}^{30-32}
$$

Similarly TDC offset between detector 17 and 32 are found using numerous paths passing through nearest neighbours and the mean value gives the precise value of TDC offset as shown in Figure 2. 


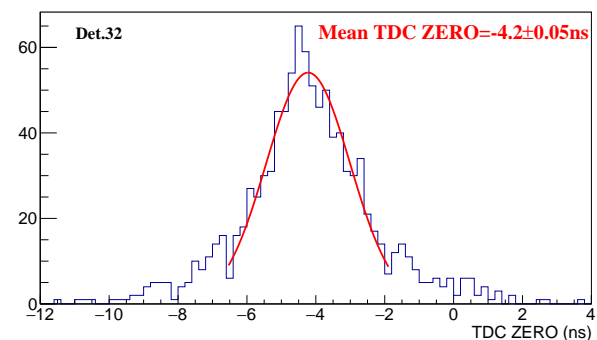

(a) TDC offset of Det.32

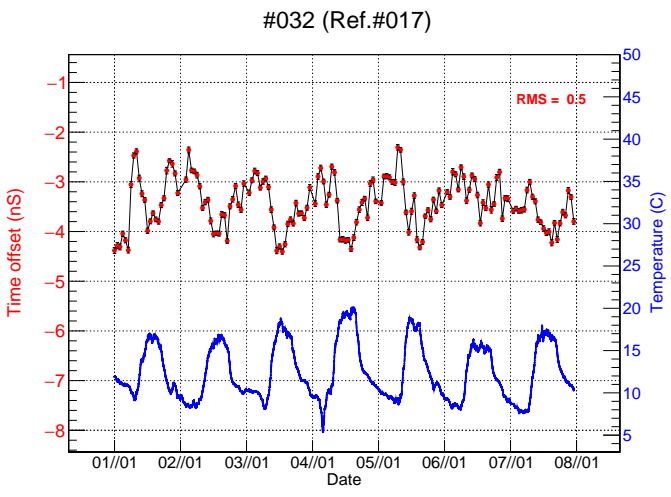

(b) Variation of TDC offset of Det.32 with temperature

Figure 2

\section{Verification of the method}

The random walk method was verified through simulations. For this pupose, about 100000 proton showers were generated for 15 energy intervals in the range of 1-1000 TeV using CORSIKA package. For hadronic interactions, SYBILL and FLUKA models were used in the high and low energy regime respectively whereas EGS package was used for electromagnetic interactions. The angular distribution of showers was uniform with their zenith angles in the range of $0-60^{\circ}$. The slope of the differential energy spectrum was taken to be -2.7. The output of CORSIKA simulation gives the arrival time and position of shower particles on a horizontal plane, along with the $\mathrm{X}$ and $\mathrm{Y}$ component of their momenta.The angle of incidence of the particles on the plane can be calculated directly from their momenta values, and since GRAPES-3 array is located on an uneven terrain, the measurement of angle of incidence of secondary particles is essential to do further corrections, as will be described later in the same section.

In CORSIKA output, the core of the shower is always at the orgin. Therefore, in order to have a more realistic approach, the core of the showers, simulated earlier, were distributed randomly over a circular area of radius $120 \mathrm{~m}$. In order to acquire lager statistics, this procedure was repeated 10 times with different seeds for the production of random numbers.Hence, in total, $10^{6}$ showers(in each energy range) were used for the analysis.

For the initial analysis, we assumed the array to be flat. Therefore if a particle's position is within the $1 \mathrm{~m}^{2}$ area constituting the detector, it implies that the particle has passed through the scintillator blocks, depositing certain amount of energy. However,the secondary $\gamma$ particles deposit only a very small amount of energy in the scintillator detectors which is below the threshold energy level for triggering the detectors, and therefore are not detected efficiently by the detector. Hence,all $\gamma$ particles were rejected, and moreover, any other particle was rejected if the energy deposited by a particle was less than half the amount of energy deposited by a single muon.

In case of simulations, the peak of $\Delta t$ distribution, and also the TDC offset of all detectors, must be ideally zero as shown in Figure 4a. After obtaining the peak value of $\Delta \mathrm{t}$ distribution (say $(\Delta t)_{p}$ ) for every neighbouring pair, random walk method is used to obtain the TDC offset of all detector 


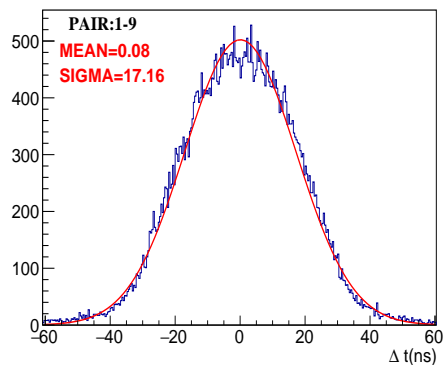

Figure 3: $\Delta t$ distribution of Pair:1-9 from simulations

with respect to a common detector. Distribution of TDC offset values for different detectors for two different energy ranges are shown in Figure 5a and Figure 5b. The mean TDC offset value of all the detectors in different energy ranges are shown in the table below. (The trigger efficiency of the GRAPES-3 is $50 \%$ at $30 \mathrm{TeV}$.Below this energy, the trigger efficiency is much lower and hence the values are quoted from $30 \mathrm{TeV}$ )
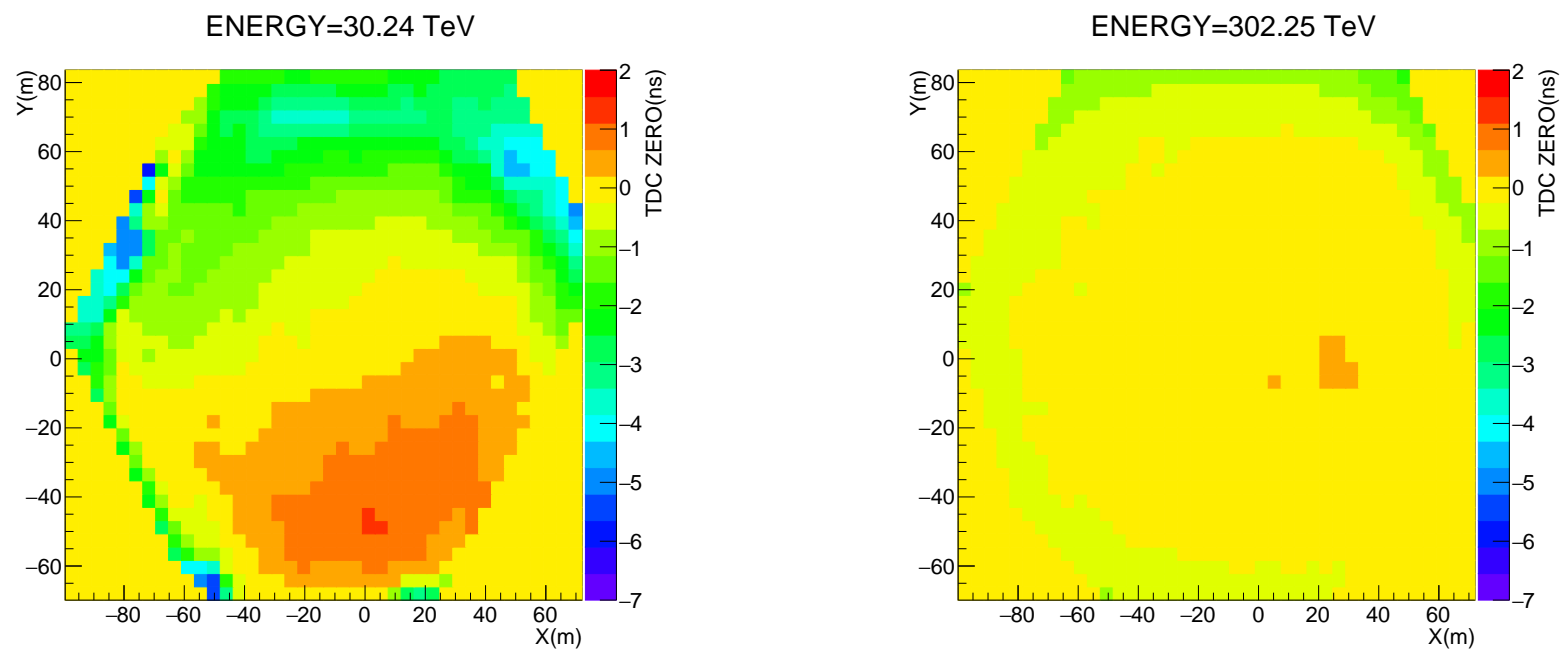

Figure 4: Contour plot showing the variation of TDC offset with position of the detector in the array

\begin{tabular}{ccc}
\hline Median Energy(TeV) & Mean(ns) & RMS(ns) \\
\hline 47.95 & -0.42 & 1.09 \\
76.01 & -0.30 & 0.61 \\
120.33 & -0.25 & 0.55 \\
190.55 & -0.30 & 0.38 \\
302.25 & -0.27 & 0.29
\end{tabular}

The larger RMS at lower energies is mainly contributed by the detectors in the outer rings as shown in Figure 5a. This is because the detectors in the outer ring do not participate in the trigger and hence lower energy showers, having smaller shower sizes, do not trigger the array even if its 
core is at a relatively short distance from the boundary of the array. Hence the $\Delta t$ distribution of neighbouring detector pairs in the outer rings are mainly dominated by showers whose cores are well within the array. Hence these pairs are mainly exposed only to peripheral part of the showers, whereas the detector pairs inside are exposed to a vaster ensemble of showers. But as energy of the primaries increase, more showers that fall outside the array are also capable of triggering the array and hence even in the outer rings $\Delta t$ peak values approch zero.

So far we assumed that the array is flat. Now, the same analysis is repeated by considering the difference in $\mathrm{Z}$ co-ordinates of the detectors. For this, intially the detectors are arranged in descending order according to its z co-ordinates. Assume that a flat surface parallel to the XY plane is holding each of the detectors. The surface holding the topmost detector is assumed to be the same horizontal plane as that in the output of CORSIKA. In this plane, we have the $\mathrm{X}$ and $\mathrm{Y}$ co-ordinates of all particles and it is checked whether any particle has passed through the topmost detector. Now, we reconstruct the path of the particles as it passes through various horizontal planes and at each plane it is checked whether any particle has passed through the detector in that particular plane. The $\mathrm{X}$ and $\mathrm{Y}$ co-ordinate of a particle at any given plane is

$$
\begin{gathered}
X=X_{i}+\Delta Z \tan \left(\theta_{p}\right) \cos \left(\phi_{p}\right) \\
Y=Y_{i}+\Delta Z \tan \left(\theta_{p}\right) \sin \left(\phi_{p}\right)
\end{gathered}
$$

where $X_{i}$ and $Y_{i}$ are the co-ordinates of the particles on the topmost plane and $\Delta Z$ corresponds to the difference in z co-ordinate of the given plane with respect to the topmost plane. Here $\theta_{p}$ and $\phi_{p}$ are the azimuthal and zenith angles of the particles which are determined from their momentum components.

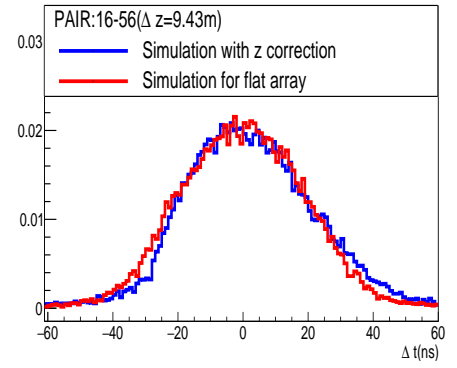

(a) $\Delta t$ distribution of pair $16-56$

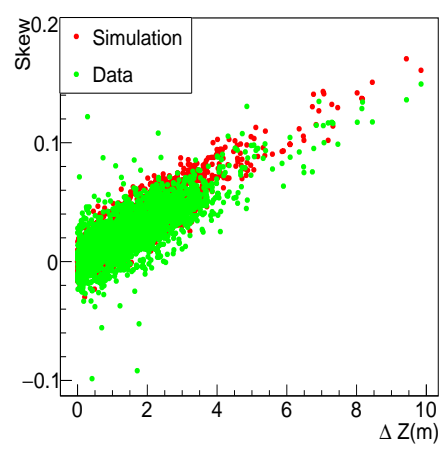

(b) Variation of skew with $\Delta Z$

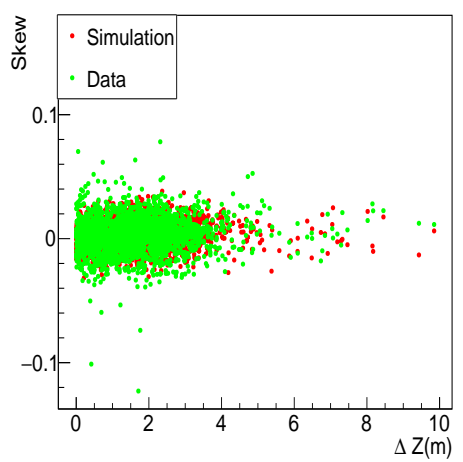

(c) Variation of skew with $\Delta Z$ (after correction)

Figure 5

After incorporating the above mentioned correction, it was observed that the $\Delta t$ distribution of some of the neighbouring pairs were asymmetric. Asymmetry was very clearly visible for detector pairs which had a large difference in heights as shown in Figure 5a. For a more vivid interpretation, the skew of all 1922 pairs of detectors were calculated and in Figure $5 \mathrm{~b}$ one can see the clear de- 
pendence of skew on the difference in height of the detectors. Even the same effects were observed in neighbouring detector distributions obtained from data.

In fact, the distribution of skew for data and simulation are coinciding as shown in Figure 6a. But when we use the more accurate formula $\Delta t+\Delta z \times \cos (\theta) \times 3.33$ for calculating effective $\Delta t$ the skew distribution coincides with the one obtained for flat array.Moreover,the correlation coefficient between skew and $\Delta Z$ reduces as is shown in Figure 5c.But,as mentioned previously, the $\theta$ values are not available before reconstruction of the shower.Hence, initially we find the direction of the shower by using the less accurate values of TDC offset calculated earlier. After obtaining the direction of shower, the correction is done for inclined showers. In this way, the asymmetry of neighbouring detector distribution disappears and more accurate values of TDC offset are obtained.

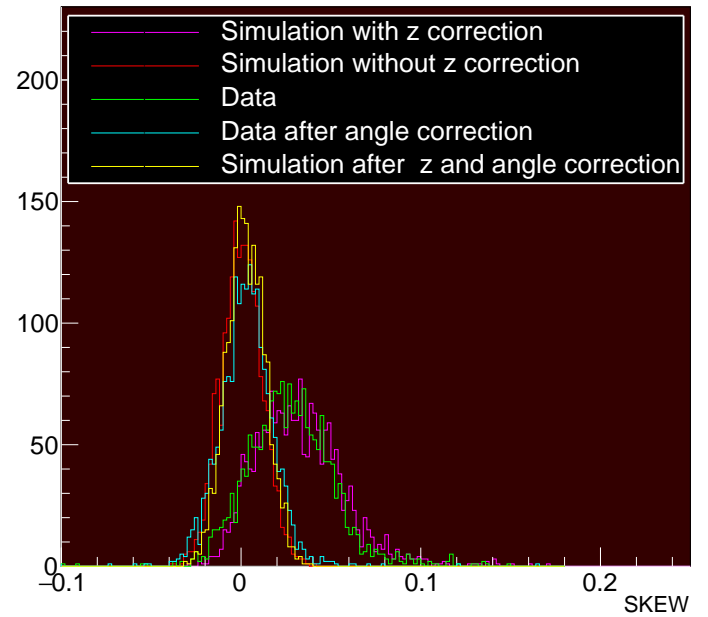

(a) Distribution of skew

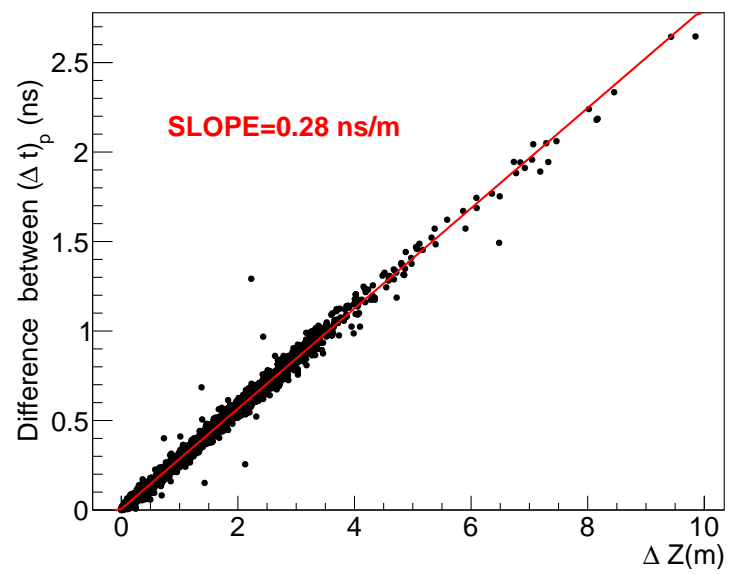

(b) Change in $(\Delta t)_{p}$ after correction

\begin{tabular}{|c|c|c|}
\hline Distribution & Mean(ns) & RMS(ns) \\
\hline Simulation without z correction & 0.002 & 0.011 \\
Simulation with z correction & 0.033 & 0.025 \\
Data & 0.027 & 0.025 \\
Data after angle correction & 0.003 & 0.015 \\
Simulation after z and angle correction & 0.003 & 0.011 \\
\hline
\end{tabular}

\section{4. conclusion}

A new technique has been developed to determine the time offset of all detectors with respect to a reference detector on an hourly basis. By taking advantage of larger statistics of shower data and small angular effect, it was possible to calculate the time offset between two neighbouring detectors with a precision of $0.3 \mathrm{~ns}$. Further on random walk method was used to calculate the time offset of all detectors with respect to reference detector. The method was validated using simulations. Before reconstruction of the shower, the direction of the shower is unknown. Hence, the TDC offset calculation is done by a two step process as mentioned previously. By using this method, we are expecting to get an improved angular resolution for GRAPES-3 array. 


\section{References}

[1] S.K. Gupta et al, GRAPES-3-A high density air-shower array for studies on the structure on the cosmic ray energy spectrum near the knee, Nuclear Instruments and Methods in Physical Research A 540(2005) 311-323

[2] P.K. Mohanty et al, Measurement of some EAS properties using new scintillator detectors developed for the GRAPES-3 experiment, Astroparticle Physics 31 (2009)24-36 